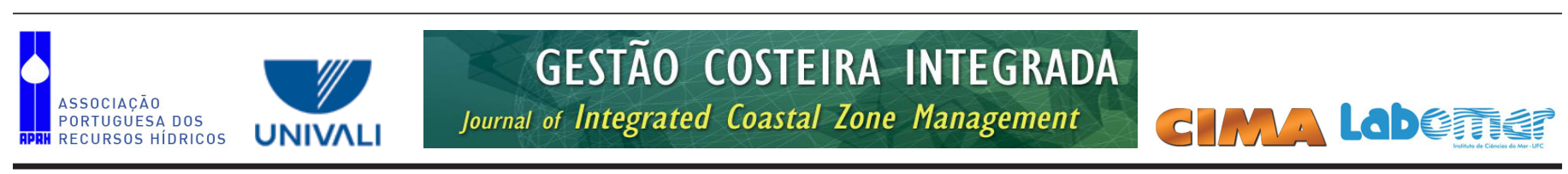

http://www.aprh.pt/rgci/pdf/rgci-429_Medeiros.pdf | D0I:10.5894/rgci429

\title{
Mapeamento acústico de areias submersas para recuperação de praias do Rio de Janeiro, Brasil *
}

\author{
Acoustic mapping of submerged sands deposits for \\ Rio de Janeiro beach nourishment, Brazil
}

\author{
Nãnashaira Medeiros ${ }^{\circledR, ~}$, , Marcelo Sperle Dias ${ }^{1}$, Artur Ayres Neto ${ }^{2}$, Dieter Muehe ${ }^{3}$
}

|

\section{RESUMO}

Devido à sua grande extensão latitudinal, a costa brasileira é influenciada por diferentes regimes climáticos e oceanográficos. Adicionalmente, a distribuição da população brasileira é caracterizada por uma alta concentraçáo nas capitais litorâneas. Todos esses fatores levam à construçáo de inúmeras estruturas de engenharia que podem, de alguma forma, impactar o transporte de sedimento e, consequentemente, o balanço sedimentar de algumas praias. Uma das formas de recuperar esse balanço sedimentar é a alimentação artificial de praias, com sedimentos provenientes da plataforma continental com características semelhantes. Os métodos geofísicos acústicos permitem mapear de forma eficiente o fundo e o subfundo marinho para a busca de áreas fontes. O objetivo deste estudo é a identificação de padróes geoacústicos, a partir de análises quantitativas e qualitativas dos tons de cinza observados nos registros sonográficos, para a caracterizaçáo de áreas fontes de areias siliciclásticas compatíveis com sedimentos de praias em erosão na cidade do Rio de Janeiro. O mapeamento foi realizado na plataforma continental interna do Rio de Janeiro, em área adjacente à praia de Itaipuaçu (Maricá, RJ). Através da análise dos registros sonográficos, de forma qualitativa e quantitativa, foi possível a identificação de 5 padróes geoacústicos distintos. Com a análise dos parâmetros sedimentológicos e de distribuição granulométrica, foram encontradas correlaçóes entre a granulometria e o selecionamento dos grãos com o sinal acústico derivado do sonar. As análises evidenciaram depósitos, localizados entre 19 e 30 metros, mais profundos que a profundidade de fechamento, em condiçóes ideais para a recomposição de praias do Rio de Janeiro. Dados complementares de sísmica rasa de alta resolução indicam que, assumindo características conservadoras, existe material suficiente para recuperar grande parte das praias urbanas do Rio de Janeiro caso venham a apresentar perda de areia, seja em decorrência de falta de aporte, seja em decorrência de elevação do nível do mar.

Palavras-chave: Oceanografia Geológica; Geofísica; Sonar de Varredura Lateral; Engorda de Praia; Geomorfologia Costeira.

\section{ABSTRACT}

The Brazilian coast is affected by different oceanographic characteristics, mainly due to its large latitudinal extension. Additionally, the distribution of the Brazilian population is characterized by a high concentration in the coastal capitals. Consequently a large number of coastal

\footnotetext{
@- Corresponding author

1 - UERJ - Universidade do Estado do Rio de Janeiro, RJ, Brasil.e-mails: Siqueira - nanashairamedeiros@gmail.com; Dias - marcelo.uerj@gmail.com

2 - UFF - Universidade Federal Fluminense, e-mail: Neto - aayres@id.uff.br

3 - UFRJ - Universidade Federal do Rio de Janeiro, e-mail: dieter.muehe@gmail.com
} 
engineering structures where constructed in these areas with impact over the sediment transport and consequently over the sediment budget. Natural processes as temporary alterations of longshore sediment transport have resulted in loss of beach sediments and the need of restoration as was the case of the Leblon Beach in Rio de Janeiro. Considering also the expected modification of climate and sea level rise the need of beach restoration will become an option to be considered. For this, the beach restoration through nourishment with sand extracted from the continental shelf is frequently the best option to consider. To find suitable sediments for dredging in the shelf, the use of geophysical methods for the mapping of the bottom and sub bottom environments represent an adequate tool. Therefore the objective of this study is the application of geophysical methods for the identification of geoacoustics patterns, following qualitative and quantitative analyzes, in order to identify suitable source areas of siliciclastic sands for the restauraration of urban beaches in Rio de Janeiro city. The mapping was done in the shelf in front of Itaipuaçu beach (Maricá, RJ). From geophysical data analysis, by quantitative and qualitative form, five geoacoustics patterns where identified which in turn where correlated with grain size parameters obtained through sieve analysis. The quantitative results showed well sorted suitable median grain size sand placers seaward of the closure depth, located between 19 and 30 meters, while reflection seismic data indicated an availability of enough material for the restoration of most of the urban ocean beaches of Rio de Janeiro.

Keywords: Geological Oceanography; Geophysics; Side Scan Sonar; Beach Nourishment; Coastal geomorphology.

\section{INTRODUÇÃO}

O litoral brasileiro tem cerca de $8.000 \mathrm{~km}$ de comprimento e, por sua vasta extensão latitudinal, sofre influências de diversos regimes climáticos. Adicionalmente, a distribuição populacional de forma irregular ao longo do litoral gera uma alta concentração da população nas grandes capitais, o que, consequentemente, traz para essas regióes um grande número de obras que impactam o balanço sedimentar litorâneo, como, por exemplo, quebra-mares, estabilização de canais de maré, acesso a terminais portuários etc. (Muehe, 2006; Neves $\&$ Muehe, 2008). Por todos esses fatores, algumas praias são impactadas e sofrem um déficit no balanço sedimentar ao longo do tempo. Uma opção para recuperação da faixa de areia das praias é a "engorda de praia" com a deposição de sedimentos de áreas fontes com características semelhantes ao sedimento local.

Publicaçóes sobre a morfodinâmica de praias do Rio de Janeiro mostram que as praias de Ipanema, Leblon, Grumari, Reserva, Prainha e Barra apresentam indícios de erosão e são compostas por areias siliciclásticas com granulometria predominantemente de areia média e baixas concentraçóes de matéria orgânica e carbonatos (Sperle et al., 1999; Kaji et al., 2006). Dados do Grupo de Pesquisa em Oceanografia Geológica (GPOG) indicam que o trecho Ipanema - Leblon tenha sofrido uma perda nos últimos anos de 1 a 2 milhóes de metros cúbicos.

Os métodos geofísicos acústicos possibilitam, de forma bastante eficaz, a busca por áreas fontes através da investigação de características geológicas e geotécnicas da superfície e subsuperfície de ambientes submersos.

O objetivo deste estudo é apresentar a identificação de padrôes geoacústicos de forma eficiente, a partir de análises qualitativas e quantitativas dos tons de cinza dos registros sonográficos, que caracterizem pacotes de áreas fontes de areias em condiçóes ideais para recuperação de praias em erosão.

Para a alimentação artificial de praias, uma das etapas mais importantes é a escolha da área fonte das areias, ou seja, identificar uma região com um volume adequado de sedimentos disponíveis para a lavra. Segundo Dean (2002), mais de $95 \%$ dos sedimentos utilizados para a alimentação artificial de praias advêm de fontes de sedimentos marinhos.
As áreas fontes devem estar localizadas relativamente próximas do seu destino final para minimizar os custos de transporte.

As áreas fontes devem dispor de uma porção disponível tanto em área quanto em volume. Geralmente, a extração ocorre em áreas de $1 \mathrm{~km}^{2}$ a $10 \mathrm{~km}^{2}$ e em profundidades na ordem de 2 a 10 metros (Dean, 2002). Porém, há de se observar as condiçóes ambientais da região de lavra para a decisão do volume de material que será extraído, para que não seja ocasionado um impacto negativo irreversível e de alta magnitude na dinâmica sedimentar na região explorada.

Os sedimentos devem ser compatíveis com a área a ser recuperada, e, preferencialmente, a regiáo deve ser livre de afloramentos rochosos por questóes operacionais. Prevendo o uso das areias para recuperação de praias cujo uso seja também recreativo, o material de alimentação deve conter um mínimo de grânulos e de argila e silte. O material para recomposição também deve ser limpo, livre de contaminantes (Dean, 2002).

Dessa forma, foi realizada uma investigação em uma região adjacente à praia de Itaipuaçu (Maricá, RJ) para a busca de áreas fontes de areias siliciclásticas compatíveis com praias em erosão no litoral da cidade do Rio de Janeiro. Cartas sedimentológicas do REVIZEE - Programa de Avaliação do Potencial Sustentável de Recursos Vivos na Zona Econômica Exclusiva (Dias et al., 2004) indicam que a regiáo possui uma cobertura de areias com textura adequada.

Não são recomendadas atividades de mineração em profundidades menores que a profundidade de fechamento, isto é, a profundidade a partir da qual a ação das ondas sobre o fundo provoca modificaçôes substanciais do perfil submarino decorrente da mobilizaçâo dos sedimentos. Dados observados por Muehe (2004) indicam o valor de $10,7 \mathrm{~m}$ como a profundidade de fechamento para a praia de Itaipuaçu. Um estudo realizado por Belligotti (2009) indicou profundidades de fechamento dos perfis de praia de Piratininga e Itacoatiara de $11,7 \mathrm{~m}$ e $13,4 \mathrm{~m}$, respectivamente.

Dragagens realizadas mesmo em maiores profundidades podem alterar a dinâmica sedimentar por meio da transformação do padrão de refração das ondas de uma regiáo, podendo causar, por exemplo, concentraçáo de energia devido à convergência das ortogonais (Komar, 1998). Dessa forma, mesmo ao tomar como uma condiçấo conservadora 
a dragagem em uma profundidade considerada teoricamente segura (maior que a profundidade de fechamento esperada para a regiáo), a modelagem de processos ondulatórios faz-se necessária para a preservação morfodinâmica da regiấo que se pretende realizar a lavra.

A área de estudo foi escolhida com base no trabalho de Medeiros (2010), que indica uma área com potencial para servir como jazida de areias médias quartzosas. Após o mapeamento por meio de métodos geofísicos de alta resolução ao longo da isóbata de $20 \mathrm{~m}$ entre Niterói e Maricá, verificou-se a existência de setores adjacentes à Praia de Itaipuaçu que poderiam ser áreas fontes de sedimentos para a recomposição de praias urbanas do Rio de Janeiro, assim como de praias de outros municípios próximos.

\section{METODOLOGIA}

Para a identificação de areias siliciclásticas compatíveis para a recuperação de praias da área de estudo, foi realizado um mapeamento geofísico por meio de sonografia de varredura lateral e ecobatimetria de uma região adjacente à praia de Itaipuaçu (Maricá, RJ) entre as isóbatas de 19 e $30 \mathrm{~m}$. Para a parametrizaçâo dos dados, foram coletadas 26 amostras de sedimento em 13 estaçóes.

Uma campanha para coleta de dados geofísicos foi realizada em outubro de 2011 com a utilização de um Ecobatímetro de Alta Precisão Raytheon Digital Modelo DE-719-CM na frequência de $200 \mathrm{kHz}$ e de um Sistema Digital de alta-resolução de Sonar de Varredura Lateral (Side Scan Sonar - SSS - Modelo 4100/560P) da Edge Tech. Esse sistema é composto por um transdutor/receptor rebocado
(Towfish 272T) que emite feixes laterais de ondas acústicas na frequência de $100 \mathrm{kHz}$. O posicionamento foi realizado por meio de um DGPS da Hemisphere GPS LLC, modelo Crescents $R-130$ com resolução submétrica.

O mapeamento foi executado por meio de quatro linhas geofísicas paralelas à praia de Itaipuaçu e complementares à linha geofísica obtida por Medeiros (2010), com cerca de nove quilômetros de extensão, $400 \mathrm{~m}$ de varredura, com uma sobreposição de $25 \mathrm{~m}$ entre a cobertura dos canais, sendo possível mapear uma área com cerca de $14 \mathrm{~km}^{2}$. Para o processamento dos dados, foram utilizados os softwares Hypack, SonarWiz.MAP, Oasis Montaj v. 7.0 (Geosoft Inc.) e ArcGis 10.

Após a elaboração de um mosaico sonográfico, foram escolhidas 13 estaçóes para a coleta de amostras de sedimento, em réplica, para parametrizaçáo dos diferentes padrōes geoacústicos (Figura 1).

As análises sedimentológicas foram realizadas no Laboratório de Oceanografia Geológica da UERJ e passaram pelos processos de secagem em estufa com temperatura média de $40^{\circ} \mathrm{C}$; pesagem em balança de alta precisão; oxidação de matéria orgânica com Peróxido de Hidrogênio $\left(\mathrm{H}_{2} \mathrm{O}_{2}\right)$; eliminação dos carbonatos com Ácido Clorídrico $(\mathrm{HCl}) ;$ e, finalmente, foram submetidas aos processos de análise granulométrica propostos por Krumbein \& Pettijohn (1938), Loring \& Rantala (1992) e Ponzi (1995). Os dados foram tratados estatisticamente por meio do programa Gradistat e foram obtidos os parâmetros estatísticos de Folk \& Ward (1957). A textura foi classificada, de acordo com o diagrama complementar de Shepard, entre cascalho, areia e lama (Shepard, 1954; Schlee, 1973).

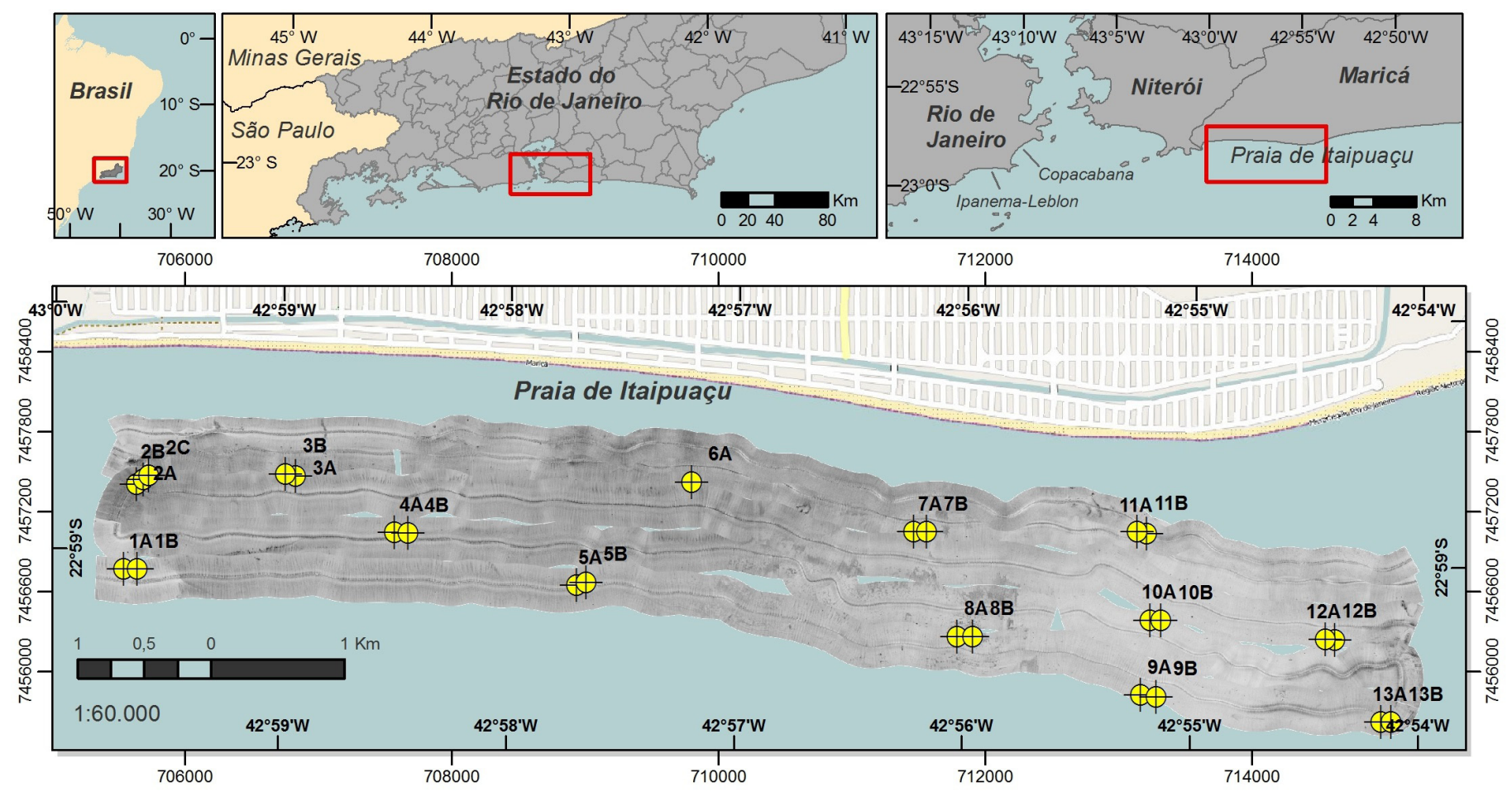

Figura 1. Mapa de localização do registro sonográfico e das amostras de sedimentos coletadas para parametrização.

Figure 1. Location map of side scan sonar profiles and sediment samples for parameterization. 
A interpretação dos registros sonográficos ocorreu por meio de análises qualitativas, da observaçáo e interpretação das feições encontradas e também por meio de uma análise quantitativa, com o software Histog, ainda em desenvolvimento. O princípio do Histog é o estabelecimento de histogramas que representam a frequência dos 256 tons de cinza presentes em pixels de uma imagem de extensão .raw no modo grayscale. Nessa escala, quanto mais próximos do número $\mathbf{0}$, mais os tons se aproximam do preto, e quanto mais próximos do número $\mathbf{2 5 5}$, mais os tons se aproximam do branco.

O objetivo do uso dessa ferramenta é obter, por meio da análise quantitativa da distribuição e frequência dos pixels da imagem sonográfica, uma classificação dos padróes geoacústicos presentes nos registros sonográficos e, assim, dispor de mais critérios para a interpretação. Foram analisadas com o Histog 13 imagens (com tamanho de $200 \mathrm{x}$ 200 pixels) que foram extraídas nas coordenadas onde foram realizadas as coletas das amostras de sedimento.

\section{RESULTADOS}

$\mathrm{Na}$ análise do mapeamento realizado na regiáo adjacente à praia de Itaipuaçu, buscou-se identificar regiôes com as características sedimentológicas adequadas para uma possível recuperação de praias em erosão no Rio de Janeiro. Como as praias em questão são de uso recreativo, as areias que forem utilizadas na recuperação devem ter características sedimentológicas semelhantes. Dessa forma, buscou-se sedimentos com granulometria de areia média, com baixos teores de matéria orgânica e carbonatos.

Para uma avaliação quantitativa dos dados sonográficos, são mostradas, na figura 2 , as curvas de frequência de tons de cinza de todas as imagens do registro sonográfico, com dimensões de $100 \mathrm{~m} \times 100 \mathrm{~m}$, referentes aos locais onde foram coletadas 13 amostras de sedimento.

A distribuição dos tons de cinza é bastante variável quando são comparados, conjuntamente, todos os 13 histogramas. Para a análise dessa distribuição, foram calculados os seguintes parâmetros estatísticos dos histogramas: o maior Número de Ocorrência de um tom de cinza (No), Moda (Mo), Média (M), Mediana (Md), Desvio Padrão (Dp), Assimetria (As), Coeficiente de Assimetria (Ca) e Curtose (C).

O Número de ocorrência indica a homogeneidade das imagens. As que possuem os maiores números de ocorrência de um tom de cinza são mais homogêneas. A Moda é o valor do tom de cinza que detém o maior número de ocorrências, indicando o valor mais representativo da imagem analisada.

A Mediana é a medida de tendência central da distribuição dos tons de cinza, ou seja, o número que separa a metade inferior da metade superior da amostra. A Média é o valor que aponta para onde mais se concentram os dados da distribuição. A distribuição da média e da mediana foram muito similares.

O Desvio Padrão representa o quanto os valores dos dados se dispersam em relação à Média. Dessa forma, observou-se que as imagens que apresentaram os valores do desvio padrão mais altos são mais heterogêneas que as outras.

A Assimetria é o grau de afastamento de uma distribuição do seu eixo de simetria, podendo ser simétrica, positiva ou negativa. Para seu cálculo, são analisadas as relaçôes entre os valores da moda, da média e da mediana. Quando esses valores são iguais, a distribuição pode ser considerada simétrica. Quando a diferença entre a Média e a Moda é maior que zero, a assimetria é classificada como positiva. Quando essa diferença é menor que zero, a assimetria é negativa.

$\mathrm{O}$ coeficiente de assimetria considera se a assimetria é moderada, quando $0,15 \leq \mid$ As $\mid<1$, ou forte, quando $\mid$ As $\mid \geq 1$,
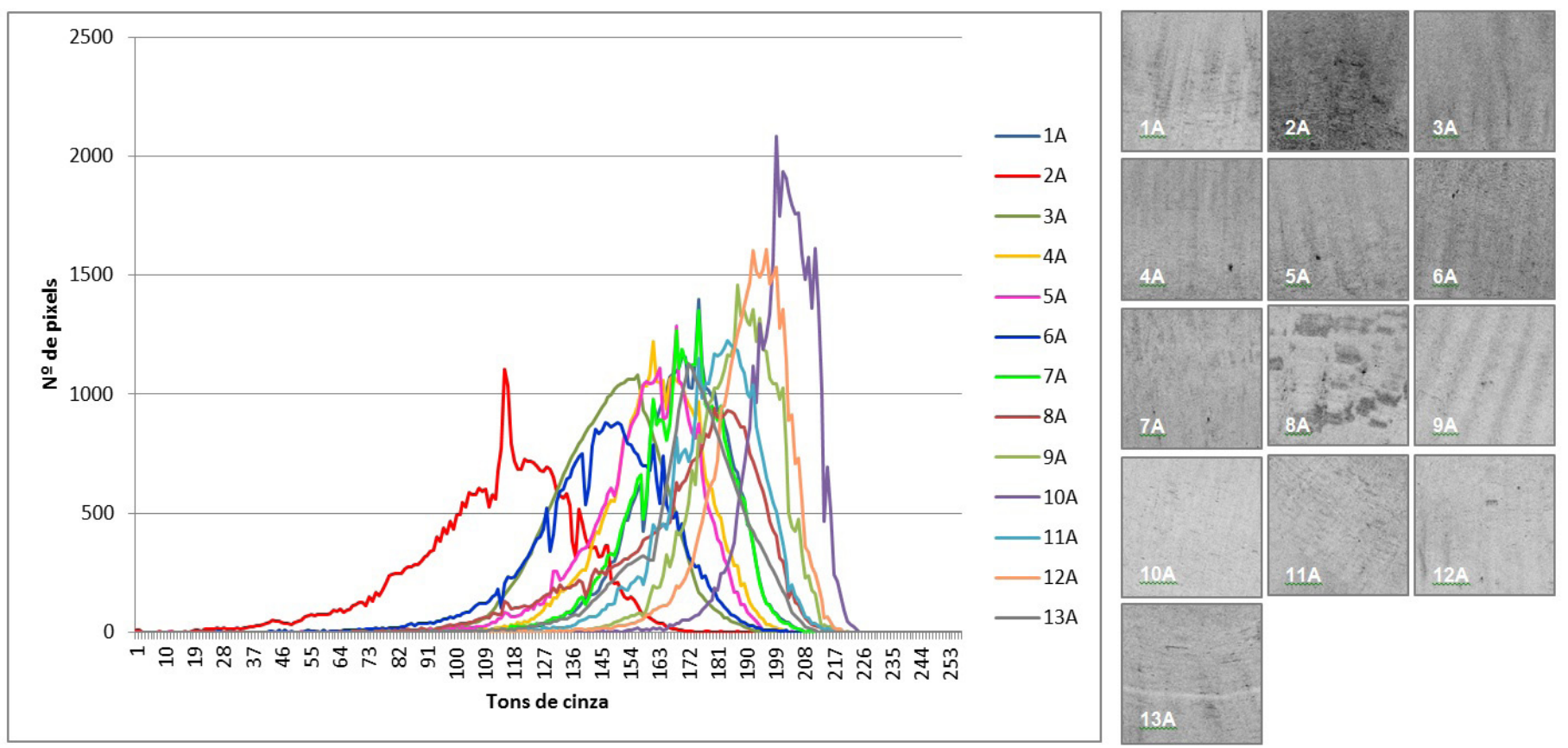

Figura 2. Distribuição dos 13 histogramas das imagens de onde foram coletadas as amostras de sedimento.

Figure 2. Distribution of the 13 histograms of the images from which sediment samples were collected. 
onde:

$$
A s=\frac{3(M-M d)}{D p}
$$

Devido à escala de tons de cinza utilizada, todas as distribuiçóes apresentaram assimetria negativa moderada. Os parâmetros de assimetria e coeficiente de assimetria não evidenciaram uma direta relação de similaridade entre os padrôes sonográficos.

A curtose indica o nível de achatamento da curva, ou seja, o grau de distribuição das frequências em relação a um parâmetro, nesse caso, os tons de cinza. Quando $C=0,263$, a curva de distribuição das frequências é mesocúrtica, quando $C>0,263$, a distribuição é platicúrtica, e quando é $\mathrm{C}<0,263$, a distribuição é leptocúrtica. Os valores da curtose variaram entre 6,02 e 25,68. Dessa forma, a distribuição dos tons de cinza de todas as curvas pode ser considerada platicúrtica. Porém, o grau de achatamento das curvas foi correlacionado à homogeneidade da distribuição.

Sendo assim, as correlaçóes existentes a respeito da distribuiçáo dos tons de cinza entre uma imagem e outra foram evidenciadas pela distribuição do número de máxima ocorrência dos tons de cinza, moda, média, mediana, desvio padrão e curtose.

Para a melhor observação das semelhanças entre os resultados estatísticos derivados da distribuição de tons de cinza das imagens, os valores dos parâmetros foram enquadrados na mesma escala (Figura 3 ).

A semelhança entre os valores observados na distribuição de cada parâmetro refletiu a concordância entre as texturas das imagens. Éimportantedestacarque, nessecaso, a denominação "textura" é empregada para descrever as diferenças visuais do registro sonográfico, que é diferente de textura do sedimento. A textura da imagem do registro sonográfico pode representar não só as características sedimentológicas, mas também as características morfológicas, como o relevo e a refletividade do fundo (backscatter).

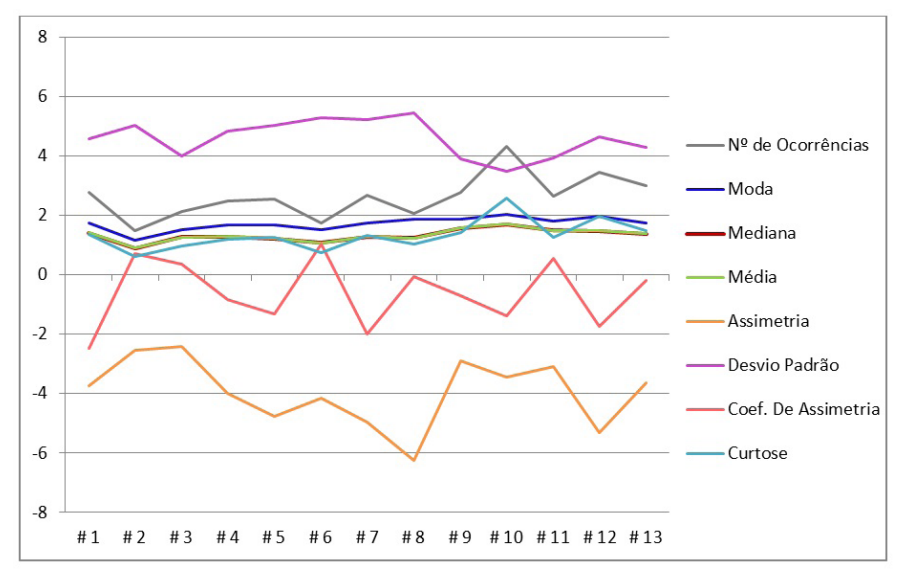

Figura 3. Distribuição dos parâmetros estatísticos referente à análise das curvas mostradas na Figura 2.

Figure 3. Distribution of statistical parameters related to the curves shown in Figure 2.
A partir da análise dos dados geofísicos, foi possível a classificação de 5 padróes de textura da imagem do registro sonográfico (Figura 4). A delimitação dos padróes foi feita de forma qualitativa, pela observaçáo de diferentes padróes de refletividade, e calibrada pela correlaçáo dos resultados dos histogramas. Posteriormente, cada um dos padróes foi detalhado com os resultados das análises sedimentológicas. A compilaçáo dos dados é apresentada nas Tabelas 1 a 5 .

A batimetria da regiáo variou de 19 a $30 \mathrm{~m}$ de profundidade. A diferença de intensidade das cores do registro sonográfico foi comparada em relação à batimetria, mas não foi encontrada uma relação direta entre a maior profundidade e a menor intensidade do sinal, ou uma maior intensidade em local do registro com maior elevação, o que seria esperado. Os indícios são de que as diferenças de intensidade do registro sonográfico sejam derivadas das características sedimentológicas, e não da variação batimétrica.

As amostras de sedimento apresentaram, como um todo, porcentagens muito baixas de matéria orgânica, entre $0,2 \%$ e $1,4 \%$ (exceto a amostra $3 \mathrm{~A}$, com $21,09 \%$ ), e de carbonatos, entre $0,5 \%$ e $6,6 \%$ (exceto a amostra $6 \mathrm{~A}$, com $14,4 \%$ ).

Ainda em relação à análise granulométrica, as amostras no geral se apresentaram como simétricas (com as exceçôes 1B e 3A, com assimetria muito negativa, indicando a presença de sedimentos grossos, e 4A, com assimetria muito positiva, indicando a presença de finos - silte e argila). No que se refere à curtose das curvas de distribuição granulométrica, as amostras se dividiram em leptocúrticas e mesocúrticas. Sendo assim, pode-se interpretar que algumas áreas possuem homogeneidade um pouco maior em relação ao tamanho dos grãos do que outras.

O padrão A é caracterizado por registros sonográficos de intensidade média e pouco heterogênea. As amostras de sedimento coletadas na área indicam um sedimento com granulometria de areia média. No setor oeste do padrão, as amostras da estação \#1 apresentaram textura de areia e o selecionamento variou de pobremente selecionado a moderadamente selecionado. Já no setor leste, as amostras da estação \#13 apresentaram textura de areia levemente cascalhosa e sedimento moderadamente bem selecionado. Apesar de possuir uma granulometria ideal para a recuperação de praias mencionadas, o padrão não é o melhor setor indicado para este destino pela presença de sedimento pobremente selecionado no setor oeste e pela textura de areia levemente cascalhosa encontrada nas duas amostras da estaçâo \#13 no setor leste (Tabela 1).

O padrão B é caracterizado por registros sonográficos de textura heterogênea e intensidade muito alta. As amostras de sedimento coletadas neste padrão possuem granulometria de areia grossa, textura de areia levemente cascalhosa e sedimento moderadamente selecionado em duas amostras, e moderadamente bem selecionado na outra amostra. Esse padrão não é recomendado para a recuperação das praias indicadas em razão dos resultados dos três parâmetros e das características originais das praias a regenerar (Tabela 2).

$\mathrm{O}$ padrão $\mathrm{C}$ caracteriza-se pelo registro sonográfico de textura homogênea e intensidade média. Foram coletadas amostras de 4 estaçóes nesse padrão. Os resultados das análises granulométricas indicam que a granulometriaéde areia média, em geral, de textura de areia a areia levemente cascalhosa e 


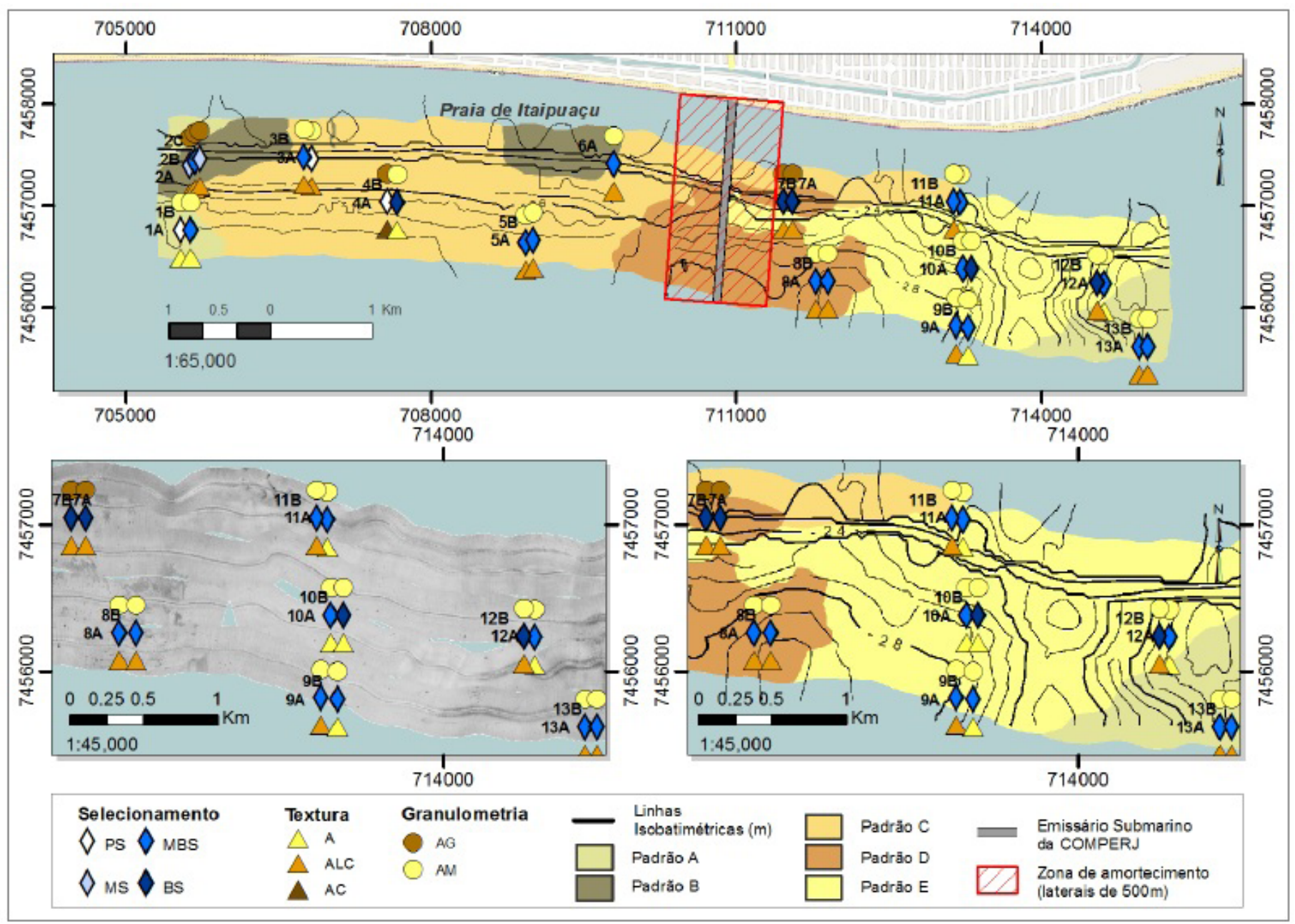

Figura 4. Mapa de classificação dos padrões geacústicos (superior) e mapas comparativos do padrão E referente ao registro sonográfico (inferior esquerda) e sua interpretação (inferior direita) com sobreposição* de parâmetros sedimentológicos relativos às amostras de sedimento coletadas para parametrizaçáo. *As amostras foram coletadas nas coordenadas geográficas onde estáo plotados os resultados do parâmetro de selecionamento.

Figure 4. Classification map of the geoacoustics patterns (top) and comparative map of the pattern E from side scan data (bottom left) and interpretation (bottom right) with overlapping * sedimentological data from sediment samples. ${ }^{*}$ The samples were collected at the same geographic coordinates of the sediment sorting values.

Tabela 1. Classificação do padrão geoacústico A em relação às imagens do registro sonográfico $1 \mathrm{~A}$ e $13 \mathrm{~A}$ e analise sedimentologica das amostras \#1 e \#13.

Table 1. Geoacoustic pattern $A$ classification from side scan sonar images $1 \mathrm{~A}$ and $13 \mathrm{~A}$ and grain size analysis of samples \# 1 and \# 13.

\begin{tabular}{|c|c|c|c|}
\hline & Padrão A & \multicolumn{2}{|l|}{ Valores } \\
\hline \multirow{3}{*}{ 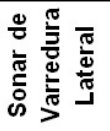 } & Intensidade & \multicolumn{2}{|l|}{ Média } \\
\hline & Textura da imagem & \multicolumn{2}{|c|}{ Heterogênea } \\
\hline & Moda & \multicolumn{2}{|c|}{$\# 1=174 ; \# 13=174$} \\
\hline \multirow{8}{*}{ 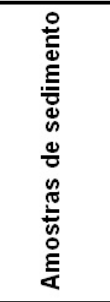 } & Estações & $\# 1$ & $\# 13$ \\
\hline & Granulometria & AM & AM \\
\hline & Faixa granulométrica $(\mu \mathrm{m})$ & 367 a 382 & 474 a 481 \\
\hline & Matéria Orgânica (\%) & 0.2 a 0.3 & 0.2 a 0.2 \\
\hline & Carbonatos (\%) & 1.6 a 1.6 & 3.9 a 4.0 \\
\hline & Selecionamento & PS a MBS & MBS \\
\hline & Grupo textural & A & ALC \\
\hline & Curtose & $M e L$ & $\mathrm{~L}$ \\
\hline
\end{tabular}

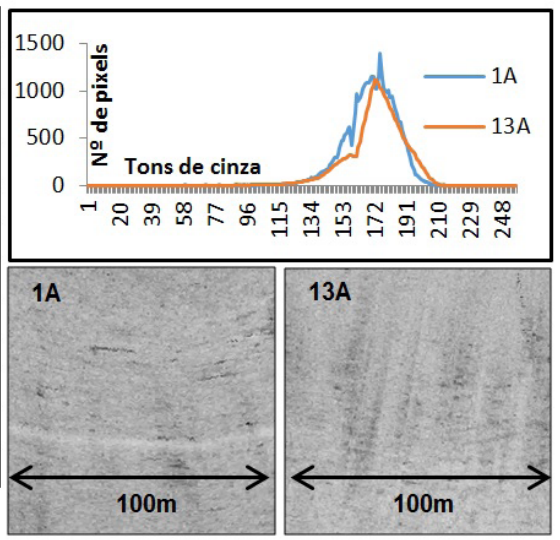

AM - Areia Média; PS - Pobremente Selecionado; MBS Moderadamente Bem Selecionado; A - Areia; ALC - Areia

Levemente Cascalhosa; M - Mesocúrtica; L - Leptocúrtica. 
Tabela 2. Classificação do padrão geoacústico $\mathbf{B}$ em relação à imagem do registro sonográfico $2 \mathrm{~A}$ e análise sedimentológica da amostra \#2.

Table 2. Geoacoustic pattern $\boldsymbol{B}$ classification from side scan sonar images $2 A$ and grain size analysis of sample \#2.

\begin{tabular}{|c|c|c|}
\hline & Padrão B & Valores \\
\hline \multirow{3}{*}{ 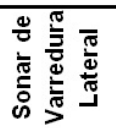 } & Intensidade & Muito Alta \\
\hline & Textura da imagem & Heterogênea \\
\hline & Moda & $\# 2-116$ \\
\hline \multirow{8}{*}{ 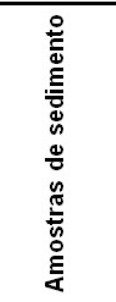 } & Estação & \#2 \\
\hline & Granulometria & $A G$ \\
\hline & Faixa granulométrica $(\mu \mathrm{m})$ & 603 a 618 \\
\hline & Matéria Orgânica (\%) & 0.2 a 0.4 \\
\hline & Carbonatos (\%) & 0.7 a 2.9 \\
\hline & Selecionamento & MS a MBS \\
\hline & Grupo textural & ALC \\
\hline & Curtose & L \\
\hline
\end{tabular}

sedimento pobremente selecionado a moderadamente bem selecionado. A estação \#4 apresentou dois resultados muito diferentes. A amostra 4A tem granulometria grossa, textura de areia cascalhosa e sedimento pobremente selecionado. Já a amostra $4 \mathrm{~B}$ apresentou areia média, com textura areia e sedimento bem selecionado. $\mathrm{O}$ padrão $\mathrm{C}$ poderia ser uma regiāo recomendada para lavra pela homogeneidade do registro sonográfico de intensidade média. Porém, as amostras de textura de areia levemente cascalhosa e a presença de sedimentos pobremente selecionados sugerem que a região não é tão adequada para recuperação de praias (Tabela 3).

$\mathrm{O}$ padrão $\mathrm{D}$ possui registro sonográfico heterogêneo de alta intensidade. As amostras coletadas são de sedimentos moderadamente bem selecionados a bem selecionados, porém são de areia média a grossa com textura de sedimentos de areia levemente cascalhosa. Apenas as informaçóes geofísicas já sugerem heterogeneidade e textura não indicadas para a recuperação de praias em erosão. Os parâmetros sedimentológicos confirmaram esse indício (Tabela 4).

Já o padrão E é caracterizado por registro sonográfico homogêneo de baixa intensidade. As amostras de sedimento indicam que a assinatura desse sinal está relacionada a sedimentos compostos por areias médias de textura areia em 5 das 8 amostras coletadas na região. As outras três são amostras de textura areia levemente cascalhosa. Além disso, o sedimento das amostras coletadas nesse padrão é bem selecionado a moderadamente bem selecionado. Essa região, consideradas as características das praias a regenerar, apresenta as condiçóes ideais para a lavra de areias quartzosas para recuperação dessas praias em erosão (Tabela 5).

Como pode ser visto na Figura 4, parte da área mapeada corresponde à regiâo onde será construído o Emissário Submarino do Complexo Petroquímico do Estado do Rio de Janeiro - COMPERJ. Um dos impactos previstos no Estudo de Impacto Ambiental do empreendimento é a restrição de atividades de exploração mineral ao longo do traçado do emissário (Petrobras, 2010). Sendo assim, mesmo considerando uma área de exclusão com laterais equidistantes de $500 \mathrm{~m}$ do emissário, a maior área correspondente ao padrão E se mantém livre.

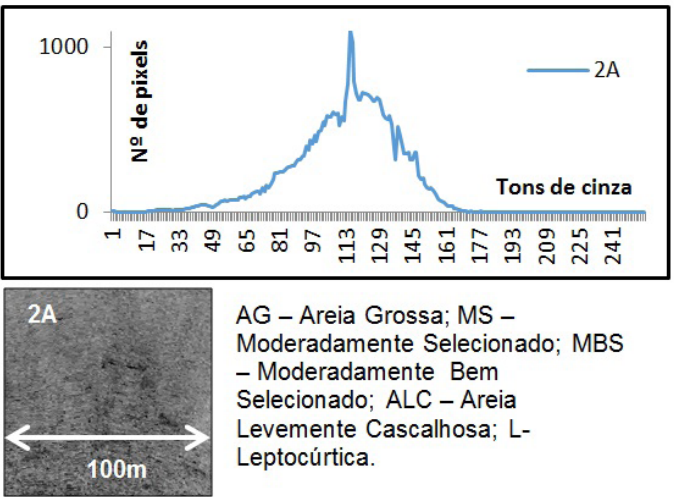

O padrão E possui uma área de $3.625 .474 \mathrm{~m}^{2}$. Registros sísmicos, realizados com perfilador de subfundo, da porção mais rasa da área de estudo (Medeiros, 2010), indicam que o pacote sedimentar aparenta ser homogêneo por pelo menos $10 \mathrm{~m}$ de profundidade, livre de afloramentos rochosos. Considerando a lavra da área do padrão $\mathrm{E}$, com 1 metro de profundidade, seria possível a extração de $3.6 \times 10^{6} \mathrm{~m}^{3}$ de areias siliciclásticas. Com 2 metros de profundidade, o volume chega a, aproximadamente, $7.3 \times 10^{6} \mathrm{~m}^{3}$. Esse volume seria mais do que o suficiente para recompor, por exemplo, o trecho Ipanema-Leblon.

\section{CONCLUSÓES}

O uso da análise quantitativa do registro sonográfico otimiza o levantamento geofísico de uma região por vários aspectos. A interpretação do mosaico sonográfico pode ser realizada de forma mais precisa e menos subjetiva. Por ser um método indireto de coleta de dados, náo dispensa a parametrizaçáo do registro sonográfico com amostras de sedimento. Permite que a parametrização seja mais eficaz, já que as estaçóes podem ser selecionadas em diferentes padróes pelo aspecto qualitativo e quantitativo. Os softwares que hoje são capazes de fornecer uma classificação automática do registro sonográfico implicam em um custo que não acompanha o grau de eficiência da classificação.

Com este estudo, foi possível verificar que setores da área de estudo possuem as características necessárias para servirem como jazidas de areias siliciclásticas para a recomposição de praias do município do Rio de Janeiro sujeitas à erosão. Assim, através da análise dos dados geofísicos, de forma qualitativa e quantitativa, foi possível a identificação de 5 padrôes geoacústicos distintos. Com a análise dos parâmetros sedimentológicos e de distribuição granulométrica, foram encontradas correlaçóes entre a textura, a granulometria e o selecionamento dos grãos com o sinal acústico derivado do sonar. Sendo assim, buscou-se padrôes de sedimentos bem selecionados, com granulometria de areia média e textura areia, como pacote sedimentar ideal para a recuperaçáo das praias, a fim de propor sedimentos que mantenham as características morfodinâmicas das praias a serem recompostas. 
Tabela 3. Classificação do padrão geoacústico $\mathbf{C}$ em relação às imagens do registro sonográfico $3 \mathrm{~A}, 4 \mathrm{~A}$, 5A e 6A e análise sedimentológica das amostras \#3, \#4, \#5 e \#6.

Table 3. Geoacoustic pattern $C$ classification from side scan sonar images $3 A, 4 A, 5 A$ and $6 A$ and grain size analysis of samples \#3, \#4, \#5 and \#6.

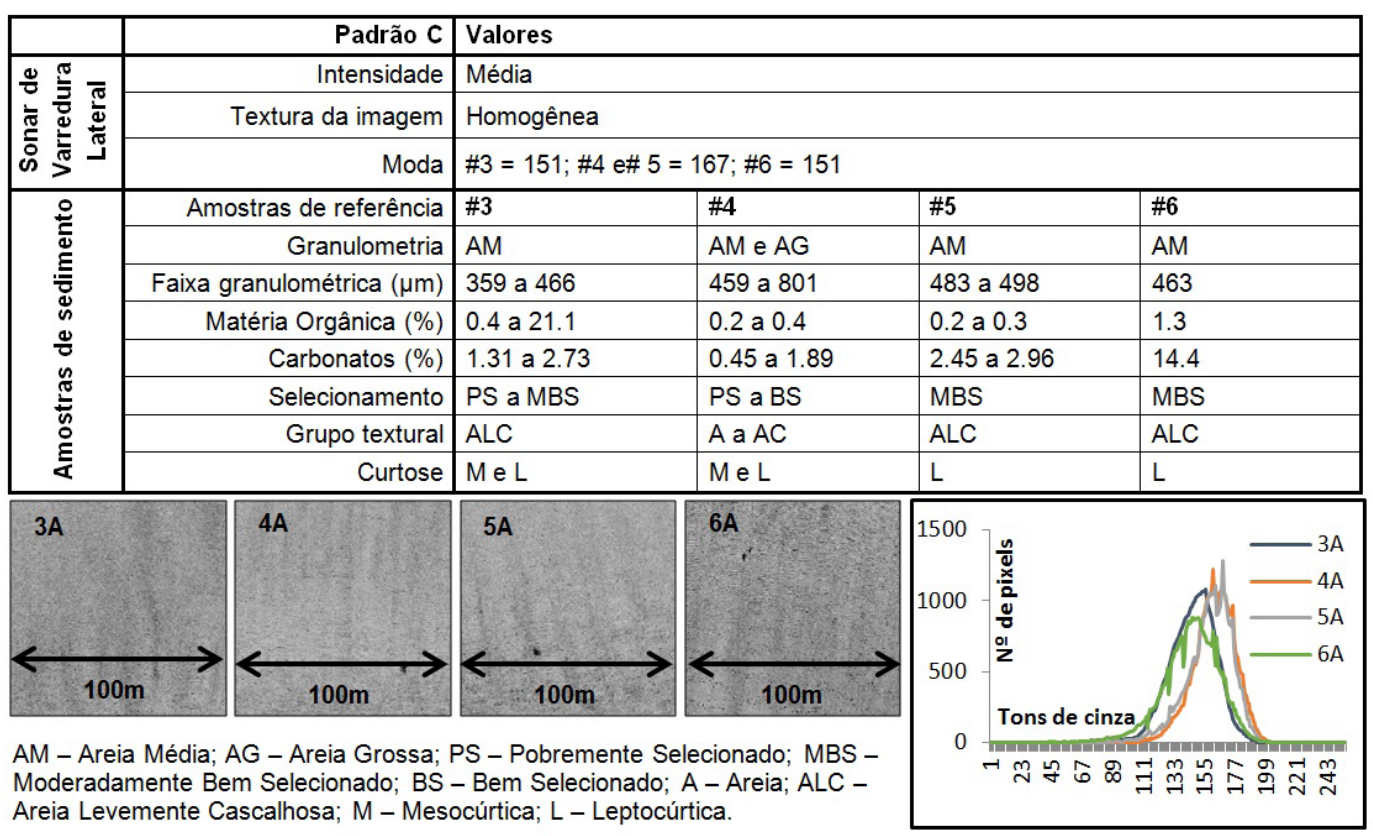

Tabela 4. Classificação do padrão geoacústico $\mathbf{D}$ em relação às imagens $7 \mathrm{~A}$ e $8 \mathrm{~A}$ do registro sonográfico e análise sedimentológica das amostras \#7 e \#8.

Table 4. Geoacoustic pattern $D$ classification from side scan sonar images $7 A$ and $8 A$ and grain size analysis of samples \#7 and \#8.

\begin{tabular}{|c|c|c|c|}
\hline & Padrão D & \multicolumn{2}{|l|}{ Valores } \\
\hline \multirow{3}{*}{ 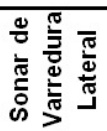 } & Intensidade & \multicolumn{2}{|l|}{ Alta } \\
\hline & Moda & \multicolumn{2}{|c|}{$\# 7=174 ; \# 8=186$} \\
\hline & Textura da imagem & \multicolumn{2}{|c|}{ Heterogênea } \\
\hline \multirow{8}{*}{ 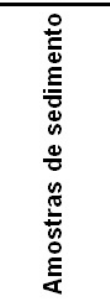 } & Estações & $\# 7$ & $\# 8$ \\
\hline & Granulometria & $A G$ & AM \\
\hline & Faixa granulométrica $(\mu \mathrm{m})$ & 507 a 523 & 462 a 465 \\
\hline & Matéria Orgânica (\%) & 0.7 a 0.3 & 0.6 a 1 \\
\hline & Carbonatos (\%) & 1.4 a 2.8 & 2.3 a 2.4 \\
\hline & Selecionamento & BS & MBS \\
\hline & Grupo textural & ALC & ALC \\
\hline & Curtose & $\mathrm{M}$ & L \\
\hline
\end{tabular}

AM - Areia Média; AG - Areia Grossa; MBS - Moderadamente. Bem Selecionado; BS - Bem Selecionado; ALC - Areia Levemente Cascalhosa; M - Mesocúrtica; L - Leptocúrtica.

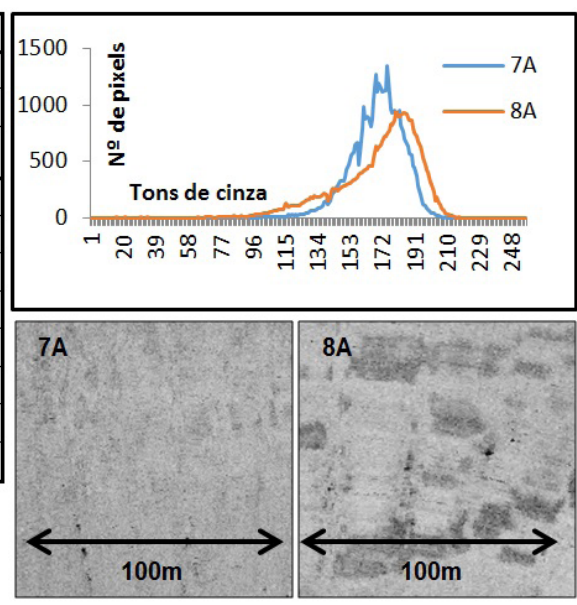


Tabela 5. Classificação do padrão geoacústico $\mathbf{E}$ em relação às imagens 9A, 10A, 11A e 12A do registro sonográfico e análise sedimentológica das amostras \#9, \#10, \#11 e \#12.

Table 5. Geoacoustic pattern E classification from side scan sonar images 9A, 10A, 11A and 12A and grain size analysis of samples \#9, \#10, \#11 and \#12.

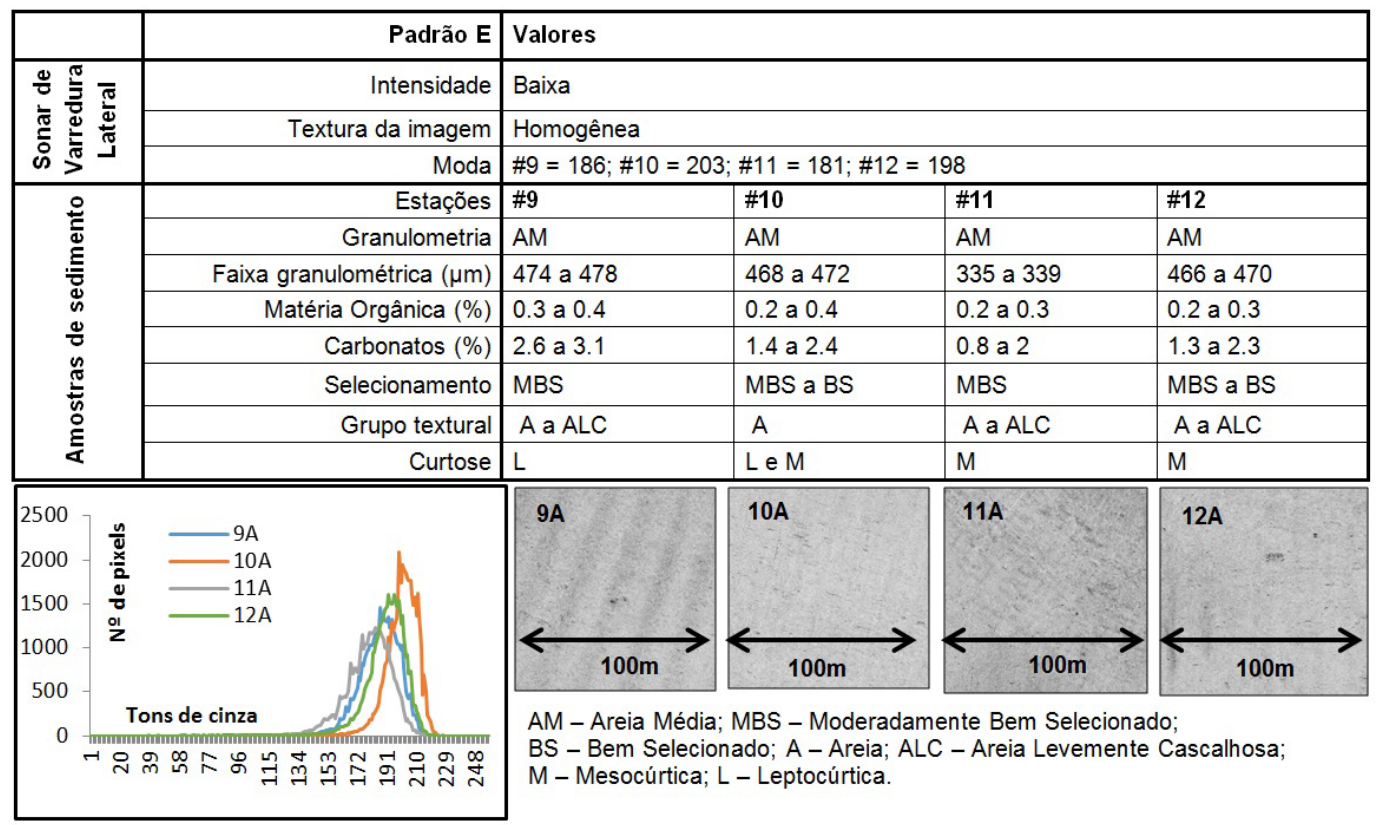

Os padrões B e D apresentaram uma maior intensidade do sinal acústico e uma heterogeneidade da imagem do registro sonográfico. Como esperado, amostras coletadas nesses padróes indicam a presença de areias grossas e textura de areia levemente cascalhosa. Os padróes A e C apresentaram uma intensidade mediana do registro sonográfico. A maioria das amostras coletadas sob esse padrão apresentaram granulometria de areia média, textura de areia levemente cascalhosa e sedimentos de pobremente selecionados a moderadamente bem selecionados.

$\mathrm{O}$ padrão $\mathrm{E}$ apresentou características muito próximas às buscadas no objetivo deste estudo. $\mathrm{O}$ sinal acústico apresentou uma intensidade baixa e uma homogeneidade na imagem do registro sonográfico. A parametrização pelas amostras indicou que esse sinal pode ser relacionado à presença de sedimentos bem selecionados a moderadamente bem selecionados, de granulometria areia média e textura areia, na sua maioria. Recomenda-se que este método seja aplicado em regióes com características similares para a busca de áreas fontes. Adicionalmente, fazem-se necessários estudos com mais métodos geofísicos para a cubagem de jazidas, amostragens de sedimentos, coletas de testemunhos para parametrização e estudos comparativos entre os sedimentos das áreas fontes e as áreas a serem recuperadas, como proposto por Oliveira e Muehe (2013).

Recomenda-se, ainda, que, junto a este método, sejam realizados estudos a respeito dos possíveis impactos ambientais sobre as comunidades bentônicas por todos os aspectos que possam afetá-las. Devem ser previstos estudos sobre a dinâmica sedimentar e caracterização bentônica tanto das áreas fontes de sedimento quanto das áreas a serem recuperadas. Dessa forma, será possível evitar a lavra sobre comunidades mais sensíveis e ameaçadas, e também verificar se grupos presentes nas áreas fontes podem representar algum risco a espécies pertencentes às áreas a serem recuperadas.

\section{AGRADECIMENTOS}

Este trabalho resultou de recursos obtidos por meio de Bolsa de Pós-Graduação da CAPES advinda do Programa de Pós-Graduação em Oceanografia da Universidade do Estado do Rio de Janeiro e de financiamentos da FAPERJ e do CNPq. Expressamos, também, os nossos agradecimentos à equipe do Laboratório de Oceanografia Geológica da UERJ.

O software Histog está ainda em desenvolvimento pelo geólogo Claudio Gino Gallea, da empresa Tethys Geofísica Ambiental, com a colaboração do Prof. Dr. Arthur Ayres Neto, docente do Programa de Pós-Graduação em Geologia e Geofísica Marinha da Universidade Federal Fluminense.

\section{REFERÊNCIAS BIBLIOGRÁFICAS}

Belligotti, F.M.(2009)-Avaliaçãometodológicadaprofundidade de convergência (profundidade de Fechamento) de perfis de três praias de energia moderada a alta no litoral do Rio de Janeiro. 130p., Dissertação de Mestrado em Geografia, Instituto de Geociências, Universidade Federal do Rio de Janeiro, Rio de Janeiro, Brasil. Unpublished.

Dean, R.G. (2002) - Beach Nourishment: theory and practice. 399p., World Scientific Publishing, Singapura..ISBN: 978-9810215484.

Dias G.T.M.; El Robrini M.; Freire G.S.F.; Figueiredo Jr., A.G. (2004) - Cartas de Sedimento de Fundo. Áreas da Oceanografia Geológica (Sul, Central, Nordeste e Norte). 
Programa de Avaliação do Potencial Sustentável dos RecursosVivos naZona Econômica Exclusiva-REVIZEE, MMA, CD-ROM de Cartas Sedimentológicas, Brasília, DF, Brasil.

Folk, R.L.; Ward, W.C. (1957) - Brazos river bar: a study in the significance of grain size parameters. Journal of Sedimentary Petrology, 27(1):3-26. Available at http://www.er.uqam.ca/ nobel/aqqua1/articles/Folk_Ward_27(1)-3.pdf

Kaji, A.; Lopes, I.T.P.; Rech, M.; Sperle, M. (2006) - Estudo da Dinâmica Sedimentar da Praia da Macumba, RJ. Simpósio Brasileiro de Oceanografia - Anais, IO-USP, São Paulo, SP, Brasil.

Komar, P.D. (1998) - Beach Processes and Sedimentation. $2^{\text {nd }}$ ed., 544p., Prentice Hall, Englewood Cliffs, NJ, U.S.A. ISBN: 9780137549382.

Krumbein, W.C.; Pettijohn, F. J. (1938) - Manual of sedimentary petrography. 549p., AppletonCentury-Crofts, New York, U.S.A. ISBN: 0918985781.

Loring, D.H.; Rantala, R.T.T. (1992) - Manual for the geochemical analyses of marine sediments and suspended particulate matter. Earth-Science Reviews, 32(4):235-283. DOI: $10.1016 / 0012-8252(92) 90001-A$

Medeiros, N.S. (2010) - Mapeamento de jazidas de areias quartzosas na plataforma continental interna do Rio de Janeiro: estudo de caso no trecho Fortaleza de Santa Cruz - Itaipuaçu. 78 p., Universidade do Estado do Rio de Janeiro, Rio de Janeiro, RJ, Brasil.

Muehe, D. (2004) - Definição de limites e tipologias da orla sob os aspectos morfodinâmico e evolutivo. Brasília. In: Ministério do Meio Ambiente e Ministério do Planejamento, Orçamento e Gestão (org.), Projeto Orla: subsídios para um projeto de gestão., pp. 11-30, Ministério do Meio Ambiente and Ministério do Planejamento, Orçamento e Gestão, Brasília, DF, Brasil. Available at: http://146.155.48.139/gestioncostera/pdf/Internacional/ Proyecto\%20ORLA\%20Brasil/volume3.pdf

Muehe, D. (2006) - Erosão e progradação no litoral brasileiro. Ministério do Meio Ambiente, Brasília, DF, Brasil. http://www.mma.gov.br/publicacoes/gestao-territorial/ category/80-gestao-costeira-g-erosao-e-progradacao

Neves, C.; Muehe, D. (2008) - Vulnerabilidade, impactos e adaptação a mudanças do clima: a zona costeira. In: Ministério da Ciência e Tecnologia (org.), Parcerias Estratégicas (ISSN: 1413-9375), pp.27: 73-108, Centro de Gestão e Estudos Estratégicos, Brasília, DF, Brasil. ISSN: 1413-9375. Available at: http://www.cgee.org.br/ parcerias/p27.php

Oliveira, F.J.; Muehe, D. (2013) - Identificação de áreas de sedimentos compatíveis na plataforma continental interna para recuperação de praias entre as cidades de Niterói e Macaé - Rio de Janeiro. Revista de Gestão Costeira Integrada / Journal of Integrated Coastal Zone Management, 13(1):89-99. DOI: 10.5894/rgci362

Petrobras (2010) - Implantação do Emissário Terrestre e Submarino do Complexo Petroquímico do Estado do Rio de Janeiro - COMPERJ. Relatório de Impacto Ambiental, INEA, Rio de Janeiro, Brasil. Available at: http://www. inea.rj.gov.br/fma/download_

Ponzi, V.R.A. (1995) - Método de análises sedimentológicas de amostras marinhas: Representação de resultados através de gráficos e mapas. Curso de Especialização em Geologia $e$ Geofisica Marinha. LAGEMAR/UFF, 51p., Niterói, Brasil. Unpublished.

Schlee, J. (1973) - Atlantic Continental Shelf and Slope of the United States sediment texture of the northeastern part. 64p., U.S. Geological Survey Professional Paper, 529-L, Washington, USA. Available at: http://pubs.usgs.gov/ pp/05291/report.pdf

Shepard, F.P. (1954) - Nomenclature based on sand-silt-clay ratios. JournalSedimentary Petrology, 24(3):151-158. DOI: 10.1306/D4269774-2B26-11D7-8648000102C1865D

Sperle, M.; Araújo, 1.; Martins, C. (1999) - Dinâmica Sedimentar em Praias Arenosas Holocênicas: Uma Proposta Metodológica. ABEQUA - Congresso da Associação Brasileira de Estudos do Quaternário, 1(7), Porto Seguro, Brasil. 